EPJ Web of Conferences 38, 09004 (2012)

DOI: $10.1051 /$ epjconf/20123809004

(C) Owned by the authors, published by EDP Sciences, 2012

\title{
Complete fusion enhancement and suppression of weakly bound nuclei at near barrier energies
}

\author{
P.R.S. Gomes ${ }^{1}$, L.F. Canto ${ }^{2}$, J. Lubian ${ }^{1}$, R. Linares ${ }^{1}$, D.H. Luong ${ }^{3}$, M. Dasgupta ${ }^{3}$, D.J. Hinde ${ }^{3}$, and M. S. Hussein ${ }^{4}$ \\ ${ }^{1}$ Instituto de Física, Universidade Federal Fluminense, Av. Litoranea s/n, Niterói, R.J. 24210-340, Brazil \\ ${ }^{2}$ Instituto de Física, Universidade Federal do Rio de Janeiro, CP 68528, Rio de Janeiro, Brazil \\ ${ }^{3}$ Department of Nuclear Physics, Research School of Physics and Engineering, Australian National University, Canberra, \\ ACT0200, Australia \\ ${ }^{4}$ Instituto de Física, Universidade de São Paulo, CP 66318, cep 05314-970, São Paulo, S.P., Brazil
}

\begin{abstract}
The influence of breakup channels on the complete fusion of weakly bound systems is investigated in terms of dynamic polarization potentials. The systematic enhancement of the cross section at sub-barrier energies seems to be consistent with recent experimental observations that nucleon transfer, often leading to breakup, is dominant compared to direct breakup at this energy regime. At energies above the barrier, the repulsive polarization potential due to direct breakup predominates and complete fusion is suppressed.
\end{abstract}

For a long time, the behavior of heavy ion fusion excitation functions has been a subject of great interest. It is widely accepted that couplings to low lying collective states may produce strong enhancement of the sub-barrier fusion cross section, particularly when nuclei with large static deformation are involved [1-6]. It has been shown that couplings to transfer channels are also important in the enhancement of sub-barrier fusion [6-16]. However, when weakly bound nuclei interact, the situation is more complex, since such nuclei have low breakup thresholds, which favors the population of states in the continuum [17-19].

A systematic study of complete and total fusion cross sections [20] uses a benchmark curve, which was called the Universal Fusion Function (UFF), given by

$F_{0}(x)=\ln [1+\exp (2 \pi x)]$

and $F(x)=\left(2 E_{c . m .} / \pi R^{2}{ }_{B} \hbar \omega\right) \sigma_{\text {fus }}$, where $x=\left(E-V_{\mathrm{B}}\right) / \hbar \omega . \mathrm{V}_{\mathrm{B}}$, $\mathrm{R}_{\mathrm{B}}$ and $\hbar \omega$ are the barrier energy, radius and curvature (parabolic barrier assumed), respectively. $\sigma_{\text {fus }}$ is the fusion cross section and $F(x)$ is called fusion function. The fusion function would be system independent if the experimental fusion cross sections were well described by Wong's formula [21]

$\sigma_{\text {fus }}{ }^{\mathrm{W}}=\left(\pi R_{B}^{2} \hbar \omega / 2 E_{\text {c.m. }}\right) \ln \left[1+\exp \left[(2 \pi)\left(E-V_{\mathrm{B}}\right) / \hbar \omega\right]\right]$.

The experimental fusion functions renormalized to take into account the failure of the Wong model for light systems at the sub-barrier energy regime and the effects of inelastic couplings $[20,22]$ are compared with UFF.
This renormalized fusion function is defined as $\bar{F}(x)=\sigma_{\exp } / \sigma_{C C} F_{o}(x)$, where $\sigma_{\exp }$ and $\sigma_{\mathrm{CC}}$ are the experimental and coupled channel fusion cross sections, respectively. If reliable potential, like double-folding potential with realistic matter densities, is used, the differences are dynamic effects due to the channels left out of the coupled channel (CC) calculations, such as breakup and transfer reactions. In the $\mathrm{CC}$ calculations, the double folding Sao Paulo potential (SPP) was used [23, 24]. This potential has been widely and successfully used in the last years, for a large variety of systems and energy ranges (for example, $[25,26]$ ), including fusion barrier distributions for weakly bound nuclei [27, 28].

Complete and total fusion (sum of complete fusion of the colliding nuclei with the fusion of part of the projectile with the target- incomplete fusion) for several systems having ${ }^{6} \mathrm{Li},{ }^{7} \mathrm{Li}$ and ${ }^{9} \mathrm{Be}$ as projectiles were compared with the UFF [20,29]. The complete fusion was found to be suppressed at energies above the barrier for all the targets investigated, in agreement with the first experimental demonstration of this effect, for the ${ }^{9} \mathrm{Be}+{ }^{209} \mathrm{Bi}$ system [30]. The quantitative dependence of suppression factor with the charge of the target nucleus has not yet been achieved [31, 32]. At sub-barrier energies, the complete fusion is enhanced in comparison with UFF. The same effect of fusion enhancement below the barrier and suppression above is observed in the reactions involving halo nuclei such as ${ }^{6} \mathrm{He},{ }^{8} \mathrm{He}$ and ${ }^{11} \mathrm{Be}$. On the other hand, total fusion of the same stable weakly bound systems were found to be unaffected by breakup and transfer couplings at energies above the barrier, for targets from ${ }^{12} \mathrm{C}$ to ${ }^{208} \mathrm{~Pb}[33,34]$. Figure 1 shows the renormalized 
complete fusion function for some of these systems. The linear scale is more appropriate to observe the effects above the barrier. The UFF curve is obtained using the Sao Paulo potential $[23,24]$. One can observe that the renormalized experimental complete fusion functions are below the UFF curve at energies above the barrier. For sub-barrier energies there is some enhancement of the complete fusion function in relation to UFF. Similarly, figure 2 shows the results for total fusion. One can observe that now there is no suppression above the barrier. So, the conclusion is that the suppression of the complete fusion is due to the incomplete fusion, which arises after the breakup of the projectile.

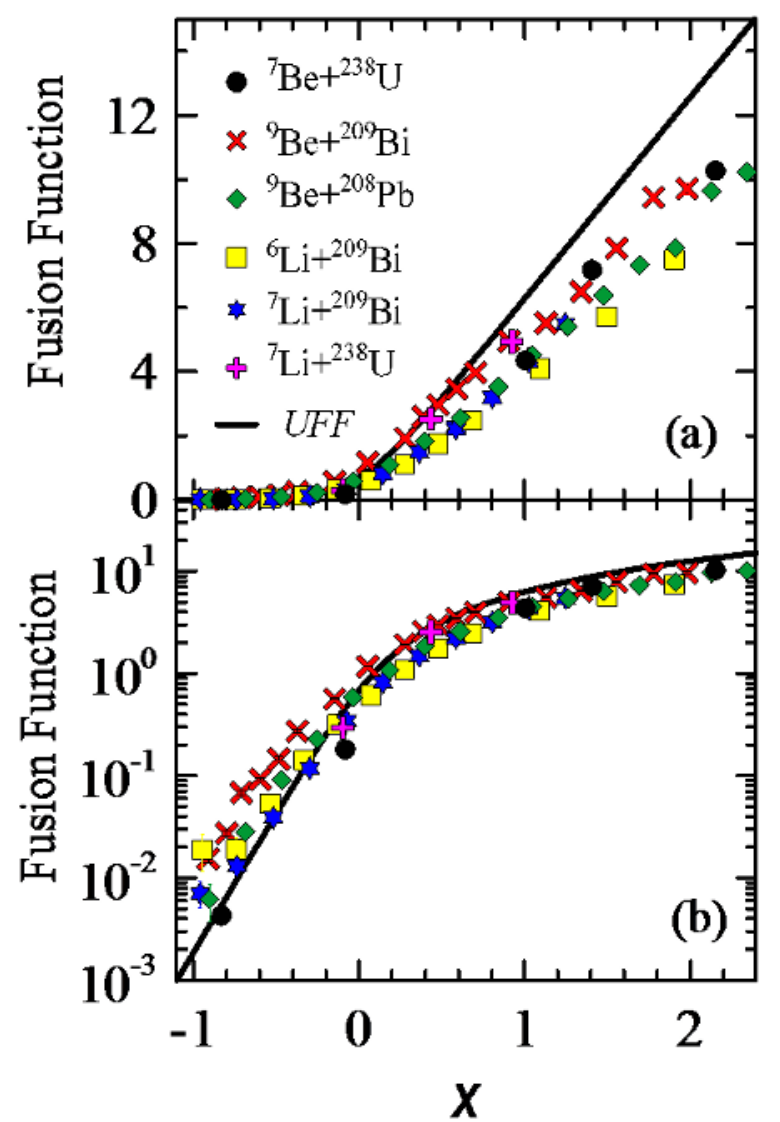

Fig. 1. Renormalized fusion functions (see the text) for complete fusion plotted against $x=\left(E-V_{\mathrm{B}}\right) / \hbar \omega$ for the several systems. The full curves are the universal fusion function (UFF) obtained by using the prescription of [20].

Although it is accepted that the CC formalism should be used for quantitative calculations, it is widely found in the literature the alternative of an energy dependent optical potential to study the effect of couplings among different reaction mechanisms. By doing so, instead of explicitly including the couplings in the $\mathrm{CC}$ formalism, the effect of couplings is manifested through an energy dependent optical potential (dynamic polarization potential-DPP). An advantage of this procedure is that the DPP can be derived from the elastic scattering measurements alone.

In the following we will try to explain these systematic behaviors of fusion cross sections in terms of dynamical polarization potentials (DPP). The systematic of the experimental fusion cross sections of weakly bound nuclei show that the couplings that lead to population of short-lived unbound or continuum states plus the transfer channels have different effects above and below the barrier. Above the barrier, the couplings that lead to prompt breakup produce fusion suppression. Below the barrier, the couplings give barrier weight at lower energies. Because of the exponential dependence of tunneling probabilities on the barrier energy, this outweighs the linear reduction in cross-section due to prompt breakup.

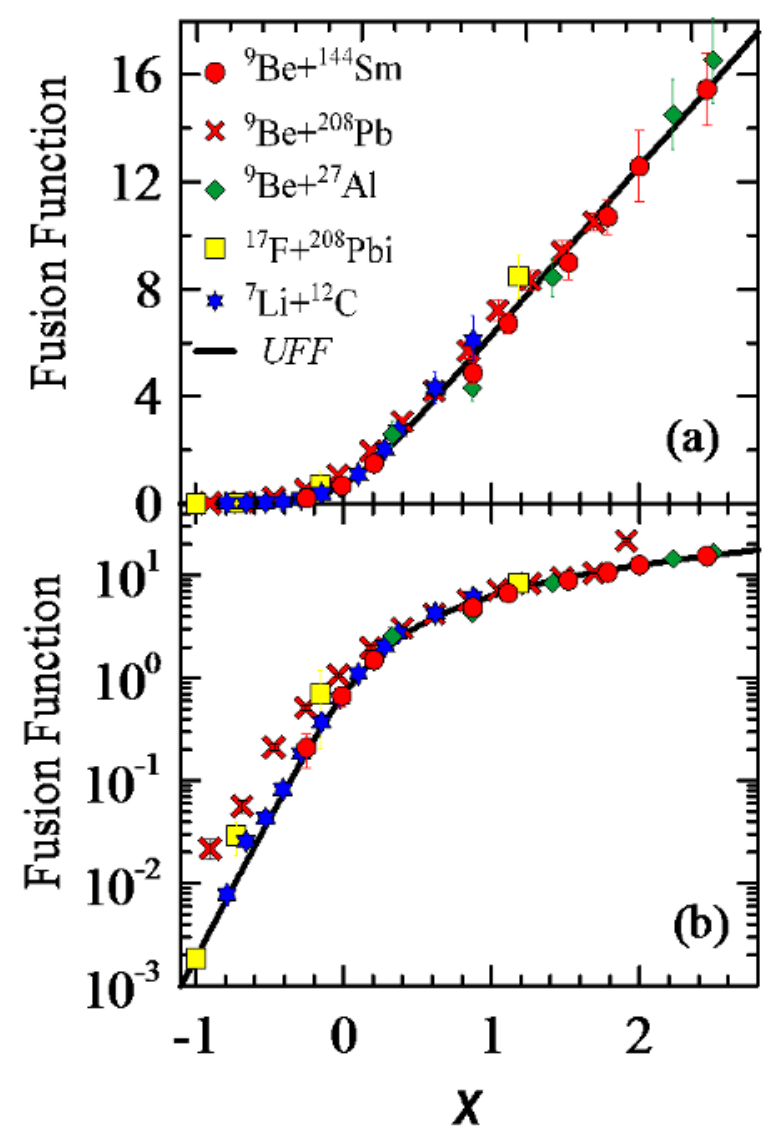

Fig. 2. Renormalized fusion functions (see the text) for total fusion plotted against $x=\left(E-V_{\mathrm{B}}\right) / \hbar \omega$ for the several systems. The full curves are the universal fusion function (UFF) obtained by using the prescription of [20].

Any polarization potential arising from couplings must be complex. The negative imaginary part accounts for the absorption of the incident wave, associated with the population of non-elastic channels. So, the incident current is attenuated as the projectile approaches the fusion barrier, reducing fusion. On the other hand, the real part of the polarization potential can be negative (attractive) or positive (repulsive), depending on the nature of the channel under consideration. Polarization potentials associated with couplings with transfer and inelastic channels were shown to be negative.

The total nuclear potential (sum of an optical potential, like folding or Akyuz-Winther, with an energy dependent polarization potential) is extracted through fits to elastic scattering data. For reactions of tightly bound nuclei, it 
exhibits the so called threshold anomaly [35-37]: as the collision energy decreases towards the barrier, the imaginary part of the potential decreases, due to the decrease of all non-elastic contributions, while the real potential shows a bell shaped maximum. This behavior comes from the polarization potential, which is attractive just below the barrier. For weakly bound systems, the energy dependence of the potential has a different behavior, since the imaginary part of the potential no longer decreases and may even increase when the bombarding energy decreases towards the barrier. This increase is accompanied by a decrease of the strength of the real part of the nuclear potential (that is, the total real potential is less attractive because the DPP is repulsive), as required by the dispersion relation. This phenomenon was called the breakup threshold anomaly (BTA) [38] and it has been attributed to the repulsive DPP produced by the breakup channel. Recently it has indeed been shown [39-41] that the direct breakup makes the real part of the DPP to become repulsive, due to the couplings among continuum breakup states (continuum-continuum couplings) [40]. The fusion cross section can be affected by both the imaginary and the real parts of the polarization potential, connected by the dispersion relation, and the general effects are discussed below.

Considering couplings to bound states, fusion can take place in the elastic or any non-elastic bound channel. So, in the calculation of the complete fusion cross section, the long range imaginary part of the polarization potential should not be used to account for the absorption of flux, but only an imaginary potential well inside the barrier, simulating IWBC. This is because the incident current absorbed from the elastic channel, due to the long range imaginary potential, goes into other channels that may also contribute to complete fusion. However, at energies below the barrier, the real part of the polarization potential is always attractive, leading to a lower fusion barrier, and consequently the complete fusion is enhanced.

Considering effects of couplings to unbound states, which may occur when the weakly bound projectile breaks up as it approaches the barrier, complete fusion requires the sequential tunneling of the fragments, which should have small probability. So, one may assume that complete fusion occurs only from the current in the non-breakup channels that reach the barrier. Consequently, the increased imaginary part of the polarization potential, due to breakup, suppresses complete fusion. So far, all the available calculations of polarization potentials for weakly bound systems consider only direct breakup and find that the real part of this potential is repulsive [3942], what makes the barrier higher, what suppress fusion.

The above discussion is not complete, because there are experimental evidences that the breakup triggered by nucleon transfer predominates over the direct breakup at sub-barrier energies [43-45]. So, the polarization potentials for each process should be evaluated separately and the results summed. The suppression of complete fusion above the barrier should result from the imaginary part of the DPP associated with direct breakup. At sub-barrier energies, the influence of the imaginary part of the direct breakup DPP on fusion should be weaker than the one of the real part of the DPP [40], which changes the barrier height. The real parts of the direct breakup and breakup after transfer polarization potentials have opposite signs. The latter is attractive while the former is repulsive. Since experimental results show that breakup after transfer is dominant, the real part of the total DPP should be negative. In this way, the fusion barrier is expected to be lower, leading to an enhancement of the complete fusion cross section, as observed in the systematic of data. Those results are also consistent with the DPP derived from elastic scattering at lower energies, as can be observed in all extrapolations to low energies of the real DPP for several systems. In those works usually there are no data at deeper sub-barrier energies, since the elastic scattering can hardly be distinguished from Rutherford scattering, but in Ref. [46] it was possible to show that DPP is attractive at this regime for the ${ }^{6} \mathrm{Li}+$ ${ }^{144} \mathrm{Sm}$ system. These explanations can be generalized for other weakly bound projectiles, like ${ }^{9} \mathrm{Be}$, for which transfer followed by breakup was measured [47], and neutron halo nuclei like ${ }^{6} \mathrm{He},{ }^{8} \mathrm{He}$ and ${ }^{11} \mathrm{Be}$, for which it has been shown that neutron transfer is more important than fusion and direct breakup at sub-barrier energies [48-52].

In conclusion, we have explained the systematic behavior found for experimental complete fusion cross sections in terms of polarization potentials. The suppression of complete fusion observed above the Coulomb barrier is attributed to the absorption of the incident flux resulting from direct breakup. The enhancement of complete fusion at sub-barrier energies is associated with the barrier lowering resulting from intermediate transfer channels. This interpretation is supported by recent experiments showing that the breakup triggered by nucleon transfer dominates the breakup cross sections at sub-barrier energies.

\section{Acknowledgments}

The authors wish to thank CNPq, FAPERJ, FAPESP, CAPES and the PRONEX for the partial financial support. The authors acknowledge the financial support of Australian Research Council Discovery Grants No. DP0879679 and No. DP110102879.

\section{References}

1. R. G. Stokstad, et al., Phys. Rev. Lett. 41, 465 (1978); Phys. Rev. C 39, 2427 (1980

2. S. Gil, et al., Phys. Rev. Lett. 65, 3100 (1990)

3. P.R.S. Gomes, et al., Phys. Rev. C 49, 245 (1994)

4. J. R. Leigh, et al., Phys. Rev. C 52, 3151 (1995)

5. D. Di Gregorio, et al., Phys. Lett. B 176, 322 (1986); Phys. Rev. C 39, 516 (1989)

6. V.V. Sargsyan, et al., Phys. Rev. C 85, 037602 (2012); Phys. Rev. C 85, 01703 (2012); Phys. Rev. C 84, 064614 (2011)

7. C. H. Dasso, S. Landowne, A. Winther, Nucl. Phys. A 405, 381 (1983); Nucl. Phys. A 407, 221 (1983) 
8. C.H. Dasso, S. Landowne, Phys. Lett. B 183, 141 (1987)

9. M. Beckerman, et al.,Phys. Rev. Lett. 45, 1472 (1980)

10. M. Beckerman, Rep. Prog. Phys. 51, 1047 (1988)

11. L. Corradi, et al., Z. Phys. A 335, 55 (1990)

12. C. R. Morton, et al., Phys. Rev. Lett. 72, 4074 (1994)

13. P.R.S. Gomes, et al., J. Phys. G: Nucl. Part. Phys. 23, 1315 (1997)

14. A. H. Wuosmaa, et al.,Phys. Lett. B 255, 316 (1991)

15. K. E. Rehm, Annu. Rev. Nucl. Part. Sci. 41, 429 (1991)

16. V.V. Sargsyan, et al., Phys. Rev. C 85, 024616 (2012); Phys. Rev. C 85, 069903 (2012); Phys. Rev. C 86, 014602 (2012)

17. L.F. Canto, P.R.S. Gomes, R. Donangelo, M.S. Hussein, Phys. Rep. 424, 1 (2006)

18. J.F. Liang, C. Signorini, Int. J. Mod. Phys. E 14, 1121 (2005)

19. N. Keeley, R. Raabe, N. Alamanos, J.L. Sida, Prog. Part. Nucl. Sci. 59, 579 (2007)

20. L.F. Canto, et al., J. Phys. G: Nucl. Part. Phys. 36, 015109 (2009); Nucl. Phys A 821, 51 (2009)

21. C. Y. Wong, Phys. Rev. Lett. 31, 766 (1973)

22. P.R.S. Gomes, et al., Phys. Lett. B 695, 320 (2011)

23. L.C. Chamon, et al., Phys. Rev. Lett. 79, 5218 (1997)

24. L.C. Chamon, et al.,Phys. Rev. C 66, 014610 (2002)

25. J.J.S. Alves, et al., Nucl. Phys. A 748, 59 (2005)

26. L. R. Gasques, L. C. Chamon, P. R. S. Gomes, J. Lubian, Nucl. Phys. A 764, 135 (2006)

27. E. Crema, L.C. Chamon, P.R.S. Gomes, Phys. Rev. C 72, 034610 (2005)

28. E. Crema, P.R.S. Gomes, L.C. Chamon, Phys. Rev. C 75, 037601 (2007)

29. P.R.S. Gomes, J. Lubian, L.F. Canto, Phys. Rev. C 79, 027606 (2009)

30. M. Dasgupta ,et al., Phys. Rev. Lett. 82, 1395 (1999)

31. P.R.S. Gomes, et al., Phys. Rev. C 84, 014615 (2011)

32. V.V. Sargsyan, et al., submitted to Phys. Rev. C

33. A. Mukherjee, et al., Phys. Lett. B 526, 295 (2002)

34. M. Dasgupta, et al., Phys. Rev. C 66, 041602(R) (2002); Phys. Rev. C 70, 024606 (2004)

35. G. R. Satchler, Phys. Rep. 55, 183 (1979); Phys. Rep. 199, 147 (1991)

36. M.E. Brandan, G.R. Satchler, Phys. Rep. 285, 143 (1997)

37. M.A. Nagarajan, C. Mahaux, G.R.Satchler, Phys. Rev. Lett. 54, 1136 (1985)

38. M.S. Hussein, P.R.S. Gomes, J. Lubian, L.C. Chamon, Phys. Rev. C 73, 044610 (2006)

39. S. Santra, et al., Phys. Rev. C 83, 034616 (2011)

40. L. F. Canto, et al., Phys. Rev. C 80, 047601 (2009)

41. J. Lubian, et al., Nucl. Phys. A 791, 24 (2007)

42. Y. Sakuragi, M. Yahiro, M. Kamimura, Prog. Theor. Phys. Suppl. 89, 136 (1989)

43. D. H. Luong, et al., Phys. Lett. B 695, 105 (2011); EPJ Web of Conf. 17, 03002 (2011)

44. A. Shrivastava, et al., Phys. Lett. B 633, 463 (2006)

45. M. Dasgupta, et al., Nucl. Phys. A 834, 147c (2010)

46. J.M. Figueira, et al., Phys. Rev. C 81, 024603 (2010)

47. R. Rafiei, et al.,Phys. Rev. C 81, 024601 (2010)

48. A. Lemasson, et al., Phys. Rev. Lett. 103, 232701

(2009)
49. R. Raabe, et al., Nature 431, 823 (2004)

50. A. Di Pietro, et al., Phys. Rev. C 69, 044613 (2004)

51. J.J. Kolata, Phys. Rev. C 71, 067603 (2005)

52. P.A. DeYoung, et al.,Phys. Rev. C 71, 051601 (R) (2005) 